\title{
Association of left ventricular ejection fraction with contrast-induced nephropathy and mortality following coronary angiography or intervention in patients with heart failure
}

\author{
This article was published in the following Dove Press journal: \\ Therapeutics and Clinical Risk Management \\ 19 July 2017 \\ Number of times this article has been viewed
}

\author{
Kun Wang ${ }^{1,2, *}$ \\ Hua-long $\mathrm{Li}^{1,2, *}$ \\ Wei-jie Bei ${ }^{1,2, *}$ \\ Xiao-sheng Guo 1,2,* \\ Shi-qun Chen ${ }^{1,2}$ \\ Sheikh Mohammed \\ Shariful Islam ${ }^{3}$ \\ Ji-yan Chen ${ }^{1,2}$ \\ Yong Liu ${ }^{1,2}$ \\ Ning Tan ${ }^{1,2}$ \\ 'Department of Cardiology, \\ Guangdong Cardiovascular Institute, \\ Guangdong Key Laboratory of \\ Coronary Disease, Guangdong \\ General Hospital, Guangdong \\ Academy of Medical Sciences, ${ }^{2}$ School \\ of Medicine, South China University \\ of Technology, Guangzhou, People's \\ Republic of China; ${ }^{3}$ The George \\ Institute for Global Health, University \\ of Sydney, Camperdown, NSW, \\ Australia \\ *These authors contributed equally \\ to this work
}

Correspondence: Ning Tan; Yong Liu Department of Cardiology, Guangdong Cardiovascular Institute, Guangdong Key Laboratory of Coronary Disease, Guangdong General Hospital, Guangdong Academy of Medical Sciences, Zhongshan Second Road, Guangzhou 510100 , Guangdong, People's Republic of China

Tel +86 I59 20172292

Fax +86208382 4369

Email gdtanning@।63.com;

liuyongmd@126.com
Background: Left ventricular ejection fraction (LVEF) is the most widely used parameter to evaluate the cardiac function in patients with heart failure (HF). However, the association between LVEF and contrast-induced nephropathy (CIN) is still controversial. Therefore, the aim of this study is to evaluate the association of LVEF with CIN and long-term mortality following coronary angiography (CAG) or intervention in patients with HF.

Methods: We analyzed 1,647 patients with HF (New York Heart Association [NYHA] or Killip class $>1$ ) undergoing CAG or intervention, including 207 (12.57\%) patients with reduced LVEF (HFrEF), 238 (14.45\%) with mid-range LVEF (HFmrEF) and 1,202 (72.98\%) with preserved LVEF (HFpEF). CIN was defined as an absolute increase of $\geq 0.5 \mathrm{mg} / \mathrm{dL}$ or a relative increase of $\geq 25 \%$ from baseline serum creatinine within $48-72 \mathrm{~h}$ after contrast medium exposure. Multivariable logistic regression and Cox proportional hazards regression analyses were performed to identify the association between LVEF, CIN and long-term mortality, respectively. Results: Overall, 225 patients (13.7\%) developed CIN. Individuals with lower LVEF were more likely to develop CIN (HFrEF, HFmrEF and HFpEF: 18.4\%, 21.8\% and 11.2\%, respectively; $P<0.001$ ), but without a significant trend after adjusting for the confounding factors (HFrEF vs HFpEF: odds ratio [OR] $=1.01$; HFmrEF vs HFpEF: OR $=1.31$; all $P>0.05$ ). However, advanced HF (NYHA class $>2$ or Killip class $>1$ ) was an independent predictor of CIN (adjusted $\mathrm{OR}=1.54,95 \%$ confidence interval $[\mathrm{CI}], 1.07-2.22 ; P=0.019)$. During the mean follow-up of 2.3 years, reduced LVEF (HFrEF group) was significantly associated with increased mortality (HFrEF vs HFpEF: adjusted hazard ratio $=2.88,95 \% \mathrm{CI}, 1.77-4.69 ; P<0.001$ ).

Conclusion: In patients with HF undergoing CAG or intervention, not worsened LVEF but advanced HF was associated with an increased risk of CIN. In addition, reduced LVEF was an independent predictor of long-term mortality following cardiac catheterization.

Keywords: cardiac catheterization, contrast-induced nephropathy, left ventricular ejection fraction, heart failure

\section{Introduction}

With the development of interventional technology and medication strategies, the number of cardiac catheterization procedures being performed continues to grow rapidly. ${ }^{1}$ Simultaneously, the incidence of contrast-induced nephropathy (CIN), a common and well-known complication which occurs following coronary angiography (CAG) or percutaneous coronary intervention (PCI) and is significantly associated with renal and cardiovascular adverse events and long-term mortality, has also increased 
gradually. ${ }^{2,3}$ Since the effective treatment measures for CIN are unknown, risk identification is important for ensuring that high-risk patients receive appropriate prophylactic measures and postoperative monitoring. ${ }^{4}$

Heart failure (HF) is a common and deteriorating condition, which has a high prevalence of ischemic origin. ${ }^{5}$ With the advancement of HF or cardiac impairment, adverse hemodynamic state results in inadequate renal perfusion and accelerates the renal impairment after contrast medium (CM) administration. ${ }^{6,7}$ Previous studies indicated that HF is one of the critical factors influencing the development of CIN. ${ }^{8,9}$ Left ventricular ejection fraction (LVEF) is another parameter that reflects the cardiac function and a useful term to categorize the type of HF, such as HF with reduced ejection fraction (HFrEF; $\mathrm{EF}<40 \%$ ), $\mathrm{HF}$ with mid-range ejection fraction (HFmrEF; $\mathrm{EF} 40 \%-49 \%$ ) and $\mathrm{HF}$ with preserved ejection fraction (HFpEF; $\mathrm{EF} \geq 50 \%$ ). ${ }^{10}$ However, the association between LVEF and the risk of CIN is still controversial. ${ }^{11-14}$ Therefore, the purpose of our study was to analyze the association of LVEF with CIN and long-term mortality following $\mathrm{CAG} / \mathrm{PCI}$ in patients with $\mathrm{HF}$.

\section{Methods}

\section{Study population}

This prospective observational study was conducted at the Guangdong General Hospital from April 2009 to December 2013. We included patients aged $>18$ years who had HF, defined as New York Heart Association (NYHA) or Killip class $>1$, and were undergoing PCI/CAG. Based on the protocol, exclusion criteria included pregnancy, malignancy, cardiovascular surgery or endovascular repair, end-stage renal disease or renal replacement, treatment with nephroprotective (eg, N-acetylcysteine) or nephrotoxic (eg, glucocorticoids, aminoglycosides) drugs and exposure to $\mathrm{CM}$ within the previous 7 days. In addition, patients who had missing preoperative or postoperative creatinine values $(n=87)$ and LVEF ( $n=448$ ) were excluded.

\section{Biochemical investigations}

Serum creatinine ( $\mathrm{SCr}$ ) concentrations were measured at admission and within 24, 48 and $72 \mathrm{~h}$ after CM administration. Other biochemical indicators were measured in the morning prior to the procedure. The Modification of Diet in Renal Disease equation was used to calculate the estimated glomerular filtration rate (eGFR), ${ }^{15}$ and the echocardiography examination was used to evaluate the LVEF. A baseline eGFR $<60 \mathrm{~mL} / \mathrm{min} / 1.73 \mathrm{~m}^{2}$ was defined as renal insufficiency. ${ }^{16}$ Furthermore, NYHA class $>2$ or Killip class $>1$ was defined as advanced HF. ${ }^{17,18}$

\section{Cardiac catheterization}

Cardiac catheterization was performed according to the standard clinical practice, by experienced interventional cardiologists. Non-ionic, low-osmolality CM was used for all patients. The type of stents was selected by the interventional cardiologists according to operative requirements. All patients received intravenous infusion of normal saline $2-12 \mathrm{~h}$ before and $6-24 \mathrm{~h}$ after the procedure at a speed of $0.5-1.0 \mathrm{~mL} / \mathrm{kg} / \mathrm{h}$. The hydration time and speed and the clinical medication were chosen based on the patient condition.

\section{Clinical end points and follow-up}

The primary end point of this study was the development of CIN, defined as an absolute increase of $\geq 0.5 \mathrm{mg} / \mathrm{dL}$ or a relative increase of $\geq 25 \%$ from baseline $\mathrm{SCr}$ level within 48-72 $\mathrm{h}$ after $\mathrm{CM}$ exposure $\left(\mathrm{CIN}_{0.5 \text { or } 25 \%}\right){ }^{19}$ Additional end point included another criteria of CIN, defined as an absolute increase of $\geq 0.3 \mathrm{mg} / \mathrm{dL}$ or a relative increase of $\geq 50 \%$ $\left(\mathrm{CIN}_{0.3 \text { or } 50 \%}\right)$ and an absolute increase of $\geq 0.5 \mathrm{mg} / \mathrm{dL}$ $\left(\mathrm{CIN}_{0.5}\right),{ }^{20}$ and all-cause mortality.

All patients included in this study were followed up by telephone or office visits at 1, 6, 12, 24 and 36 months after discharge. Adverse events were recorded on the case report form.

This study was performed according to the Declaration of Helsinki, and the ethics committee of the Guangdong General Hospital approved the study protocol. Written informed consent was obtained from the patients involved in the study.

\section{Statistical analysis}

Patients were divided into three groups based on the level of LVEF according to the 2016 European Society of Cardiology guideline for HF. ${ }^{10}$ For continuous variables, ANOVA was used for normally distributed data (described as mean \pm standard deviation), and Wilcoxon rank-sum test was conducted for non-normal distributions (described as interquartile range). For categorical variables, $\chi^{2}$ test or Fisher's exact test was used (described as absolute values and percentages). Multivariable logistic regression and Cox proportional hazards regression analyses were performed to identify the association of LVEF with CIN and long-term mortality, respectively. HFpEF was considered as the reference group. The effect of HFmrEF and HFrEF on outcomes was estimated and was compared with the reference group. Kaplan-Meier method was used to describe the all-cause mortality by log-rank tests. All statistical analyses were performed with SPSS software version 22.0 (IBM Corporation, Armonk, NY, USA) and R software (version 3.1.2; R Core 
Team, Vienna, Austria). A two-tailed $P<0.05$ was considered statistically significant.

\section{Results}

\section{Baseline demographics and characteristics}

A total of 1,647 patients with HF undergoing CAG/PCI were analyzed, including 207 (12.57\%) patients with HFrEF, 238 (14.45\%) with HFmrEF and 1,202 (72.98\%) with HFpEF. The baseline demographics and characteristics of patients are listed in Table 1.
Compared to the patients with $\mathrm{HFpEF}$, patients with HFrEF were more likely to have advanced HF, renal insufficiency and prior myocardial fraction. Furthermore, those in the HFrEF group had lower systolic blood pressure on admission and were less likely to have a history of hypertension. However, age, gender, smoking, hyperlipidemia and history of coronary artery bypass grafting were similar among the three groups.

On admission, patients with HFrEF had higher SCr and $\mathrm{N}$-terminal pro-brain natriuretic peptide concentrations, but

Table I Baseline characteristics according to the left ventricular ejection fraction group

\begin{tabular}{|c|c|c|c|c|}
\hline Variables & HFrEF (N=207) & HFmrEF $(\mathrm{N}=238)$ & HFpEF $(\mathrm{N}=\mathrm{I}, 202)$ & $P$-values \\
\hline \multicolumn{5}{|l|}{ Demographics } \\
\hline Age (years) & $64.20 \pm 10.75$ & $65.05 \pm \mid I .44$ & $64.90 \pm 10.52$ & 0.651 \\
\hline Female (\%) & $43(20.8)$ & $5 \mathrm{I}(2 \mathrm{I} .4)$ & $319(26.5)$ & 0.078 \\
\hline $\mathrm{SBP}(\mathrm{mmHg})$ & $122.62 \pm 20.49$ & $123.99 \pm 20.65$ & $|32.50 \pm 20.6|$ & $<0.001$ \\
\hline $\mathrm{DBP}(\mathrm{mmHg})$ & $76.65 \pm 12.03$ & $73.98 \pm 12.80$ & $76.16 \pm 11.78$ & 0.023 \\
\hline Advanced HF, n (\%) & $115(55.6)$ & $115(48.3)$ & $27 \mid(22.5)$ & $<0.001$ \\
\hline \multicolumn{5}{|l|}{ Medical history, n (\%) } \\
\hline Smoking & $84(40.6)$ & $106(44.5)$ & $442(36.8)$ & 0.062 \\
\hline Hypertension & $98(47.3)$ & $136(57.1)$ & $775(64.5)$ & $<0.001$ \\
\hline Diabetes mellitus & $57(27.5)$ & $64(26.9)$ & $311(25.9)$ & 0.854 \\
\hline Hyperlipidemia & $26(12.6)$ & $26(10.9)$ & $175(\mid 4.6)$ & 0.286 \\
\hline Prior MI & $44(21.3)$ & $55(23.1)$ & $103(8.6)$ & $<0.001$ \\
\hline Prior CABG & $2(1.0)$ & $3(1.3)$ & $12(1.0)$ & 0.931 \\
\hline Renal insufficiency & $71(34.3)$ & $75(31.5)$ & 239 (19.9) & $<0.001$ \\
\hline \multicolumn{5}{|l|}{ Laboratory index } \\
\hline $\mathrm{SCr}(\mu \mathrm{mol} / \mathrm{L})$ & $|07.50 \pm 46.4|$ & $101.84 \pm 37.05$ & $91.65 \pm 33.06$ & $<0.001$ \\
\hline eGFR $\left(\mathrm{mL} / \mathrm{min} / \mathrm{l} .73 \mathrm{~m}^{2}\right)$ & $70.88 \pm 25.99$ & $73.39 \pm 26.11$ & $79.79 \pm 25.01$ & $<0.001$ \\
\hline NT-proBNP (pg/mL) & $5,050.16 \pm 6,454.23$ & $2,844.92 \pm 4,20 \mathrm{I} .42$ & $\mathrm{I}, 108.34 \pm 3,42 \mathrm{I} .70$ & $<0.001$ \\
\hline LVEF (\%) & $32.01 \pm 5.74$ & $44.49 \pm 3.00$ & $63.48 \pm 6.94$ & $<0.001$ \\
\hline $\mathrm{TG}(\mathrm{mmol} / \mathrm{L})$ & $1.3 \mathrm{I} \pm 0.82$ & $\mathrm{I} .4 \mathrm{I} \pm 0.69$ & $1.55 \pm 1.25$ & 0.072 \\
\hline $\mathrm{CHO}(\mathrm{mmol} / \mathrm{L})$ & $4.29 \pm 1.05$ & $4.50 \pm 1.11$ & $4.39 \pm 1.16$ & 0.361 \\
\hline LDL (mmol/L) & $2.57 \pm 0.84$ & $2.8 I \pm 0.94$ & $2.6 I \pm 0.95$ & 0.071 \\
\hline $\mathrm{HDL}(\mathrm{mmol} / \mathrm{L})$ & $0.94 \pm 0.29$ & $0.87 \pm 0.25$ & $1.03 \pm 2.47$ & 0.721 \\
\hline $\mathrm{HbAlc}(\%)$ & $6.74 \pm 1.38$ & $6.80 \pm 1.60$ & $6.55 \pm 1.31$ & 0.030 \\
\hline HGB $(g / L)$ & $|32.4| \pm \mid 7.98$ & $131.17 \pm 17.65$ & $131.50 \pm 16.56$ & 0.749 \\
\hline Anemia, n (\%) & $67(32.4)$ & $94(39.5)$ & $420(34.9)$ & 0.262 \\
\hline \multicolumn{5}{|c|}{ Perioperative medications, n (\%) } \\
\hline ACEI/ARB & I8I (87.4) & $206(86.6)$ & $\mathrm{I}, 053(87.6)$ & 0.905 \\
\hline$\beta$-blockers & I57 (75.8) & $198(83.2)$ & I,015 (84.4) & 0.009 \\
\hline Statins & $192(92.8)$ & $230(96.6)$ & $\mathrm{I}, 160(96.5)$ & 0.033 \\
\hline Diuretics & $116(56.0)$ & $97(40.8)$ & $176(14.6)$ & $<0.001$ \\
\hline \multicolumn{5}{|c|}{ Angiographic and procedural characteristics } \\
\hline Emergent $\mathrm{PCl}, \mathrm{n}(\%)$ & $36(17.4)$ & $64(26.9)$ & $126(10.5)$ & $<0.001$ \\
\hline $\mathrm{CM}$ volume $(\mathrm{mL})$ & $124.30 \pm 69.76$ & $|40.1| \pm 69.99$ & $133.84 \pm 66.49$ & 0.046 \\
\hline $\mathrm{CM}$ volume $>100 \mathrm{~mL}$ & $101(48.8)$ & $144(60.5)$ & $687(57.2)$ & 0.034 \\
\hline Stents length (mm) & $32.69 \pm 36.07$ & $39.91 \pm 37.48$ & $37.22 \pm 33.02$ & 0.078 \\
\hline Hydration volume $\mathrm{mL}$ & $855.19 \pm 536.83$ & $894.70 \pm 492.87$ & $801.85 \pm 464.86$ & 0.014 \\
\hline Mehran risk score & $8.76 \pm 6.09$ & $8.85 \pm 5.62$ & $5.86 \pm 4.33$ & $<0.001$ \\
\hline
\end{tabular}

Abbreviations: $\mathrm{HFrEF}$, heart failure with reduced ejection fraction; HFmrEF, heart failure with mid-range ejection fraction; HFpEF, heart failure with preserved ejection fraction; SBP, systolic blood pressure; DBP, diastolic blood pressure; HF, heart failure; MI, myocardial infarction; CABG, coronary artery bypass grafting; SCr, serum creatinine; eGFR, estimated glomerular filtration rate; NT-proBNP, N-terminal pro-brain natriuretic peptide; LVEF, left ventricular ejection fraction; TG, triglyceride; CHO, cholesterol; LDL, low-density lipoprotein; HDL, high-density lipoprotein; HbA Ic, hemoglobin A lc; HGB, hemoglobin; ACEl, angiotensin-converting enzyme inhibitor; ARB, angiotensin receptor blocker; $\mathrm{PCl}$, percutaneous coronary intervention; $\mathrm{CM}$, contrast medium. 
lower eGFR and LVEF level. In addition, those patients were more likely to be on diuretics and less likely to be on $\beta$-blockers and stains than the other two groups. Furthermore, the prevalence of emergency PCI and the volume of CM were highest in the patients with HFmrEF.

\section{Incidence of CIN and in-hospital outcomes}

Overall, 225 patients (13.7\%) developed CIN, and the incidence of $\mathrm{CIN}_{0.5 \text { or } 25 \%}$ was different among the LVEF groups (HFrEF, HFmrEF and HFpEF: $18.4 \%, 21.8 \%$ and $11.2 \%$, respectively; $P<0.001)$. Similar trend was observed in the incidence of $\mathrm{CIN}_{0.3 \text { or } 50 \%}$ or $\mathrm{CIN}_{0.5}$ (Table 2 and Figure 1).

Furthermore, patients with HFrEF were more likely to experience death (HFrEF, HFmrEF and HFpEF: 5.8\%, 5.5\% and $1.0 \%$, respectively; $P<0.001$ ) and hypotension (HFrEF, HFmrEF and HFpEF: $11.1 \%, 9.2 \%$ and $2.1 \%$, respectively; $P<0.001)$. In addition, patients with lower LVEF had a significantly higher rate of requirement of intra-aortic balloon pump (IABP) (HFrEF, HFmrEF and HFpEF: 14.0\%, 12.2\% and $2.9 \%$, respectively; $P<0.001)$ and renal replacement therapy (HFrEF, HFmrEF and HFpEF: 3.4\%, 2.5\% and $0.7 \%$, respectively; $P=0.002$ ) (Table 2 ).

\section{Association of LVEF with CIN}

After adjusting for the confounders, including age $>75$ years, hypertension, diabetes mellitus, renal insufficiency, advanced $\mathrm{HF}$, prior myocardial infarction, emergency PCI, CM volume $>100 \mathrm{~mL}$, hypotension and use of stains, diuretics and IABP, multivariate logistic regression results revealed that individuals with lower LVEF were not at significantly increased risk of CIN compared with the highest LVEF group (HFrEF vs HFpEF: odds ratio [OR] $=1.01$, 95\% confidence interval [CI], 0.69-1.74; $P=0.700 ;$ HFmrEF vs

Table 2 Incidence of CIN and in-hospital outcomes between left ventricular ejection fraction groups

\begin{tabular}{|c|c|c|c|c|}
\hline Variables, $\mathbf{n}(\%)$ & $\begin{array}{l}\text { HFrEF } \\
(\mathrm{N}=207)\end{array}$ & $\begin{array}{l}\text { HFmrEF } \\
(\mathrm{N}=238)\end{array}$ & $\begin{array}{l}\text { HFpEF } \\
(\mathrm{N}=\mathrm{I}, 202)\end{array}$ & $P$-value \\
\hline $\mathrm{CIN}_{0.5 \text { or } 25 \%}$ & $38(18.4)$ & $52(21.8)$ & $135(1 \mid .2)$ & $<0.001$ \\
\hline $\mathrm{CIN}_{0.3 \text { or } 50 \%}$ & $29(14.0)$ & $38(16.0)$ & $78(6.5)$ & $<0.001$ \\
\hline $\mathrm{CIN}_{0.5}$ & $16(7.7)$ & $22(9.2)$ & $37(3.1)$ & $<0.001$ \\
\hline Death & & & $12(1.0)$ & $<0.001$ \\
\hline Hypotension & $23(11.1)$ & $22(9.2)$ & $25(2.1)$ & $<0.001$ \\
\hline Intra-aortic balloon pump & $29(14.0)$ & $29(12.2)$ & $35(2.9)$ & $<0.001$ \\
\hline Renal replacement therapy & $7(3.4)$ & $6(2.5)$ & $9(0.7)$ & 0.002 \\
\hline Cerebrovascular events & $2(1.0)$ & $3(1.3)$ & $7(0.6)$ & 0.485 \\
\hline
\end{tabular}

Abbreviations: CIN, contrast-induced nephropathy; HFrEF, heart failure with reduced ejection fraction; HFmrEF, heart failure with mid-range ejection fraction; $\mathrm{HFpEF}$, heart failure with preserved ejection fraction.

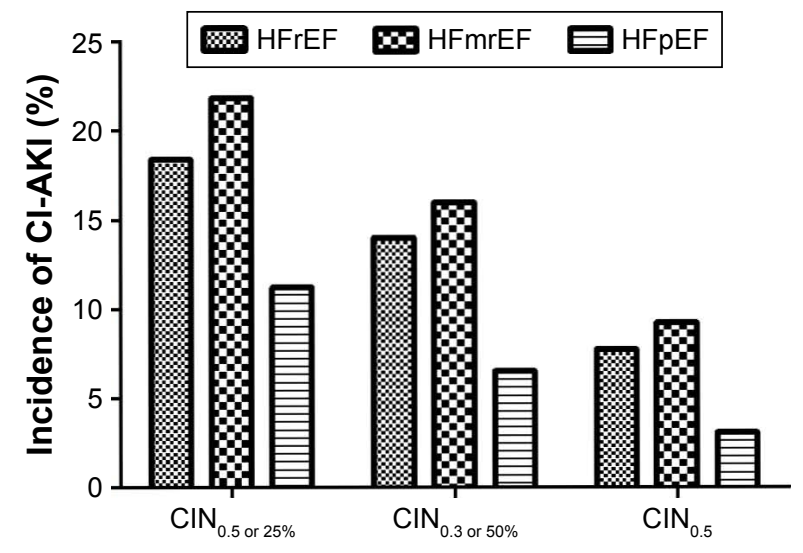

Figure I Incidence of CIN in different definitions between left ventricular ejection fraction groups.

Abbreviations: CIN, contrast-induced nephropathy; HFrEF, heart failure with reduced ejection fraction; HFmrEF, heart failure with mid-range ejection fraction; $\mathrm{HFpEF}$, heart failure with preserved ejection fraction; $\mathrm{Cl}-\mathrm{AKI}$, contrast-induced acute kidney injury.

HFpEF: OR $=1.31,95 \%$ CI, 0.87-1.96; $P=0.194)$. Similar results were demonstrated for $\mathrm{CIN}_{0.3 \text { or } 50 \%}$ or $\mathrm{CIN}_{0.5}$. However, age $>75$ years, advanced HF, emergency PCI and use of IABP were the significantly independent risk factors for CIN in different criteria (Table 3).

\section{Association between LVEF, CIN and long- term mortality}

The mean follow-up period was $2.30 \pm 0.93$ years. Log-rank analyses indicated that patients with lower LVEF were associated with higher mortality rate (log-rank, $P<0.001)$. The Kaplan-Meier curve is shown in Figure 2. After adjusting for the confounders which were associated with longterm mortality, multivariate Cox regression showed that HFrEF was an independent predictor of mortality (HFrEF vs HFpEF: adjusted hazard ratio $[\mathrm{HR}]=2.88,95 \% \mathrm{CI}$, 1.77-4.69; $P<0.001$; HFmrEF vs HFpEF: HR $=1.55,95 \%$ CI, 0.95-2.53; $P=0.079$ ) (Table 4).

Moreover, patients who developed $\mathrm{CIN}_{0.5 \text { or } 25 \%}$ had higher rate of all-cause mortality than those without during the follow-up. Similar results were found in those who developed $\mathrm{CIN}_{0.3 \text { or } 50 \%}$ or $\mathrm{CIN}_{0.5}$ (Figure 3).

\section{Discussion}

To our knowledge, this is the first study to describe the clinical characteristics and investigate the association of LVEF with CIN and long-term mortality following CAG/PCI in patients with HF. Our data showed that patients with lower LVEF were more likely to have comorbidities and develop CIN. However, advanced HF was significantly associated with an increased risk of CIN. In addition, age $>75$ years, 
Table 3 Association of left ventricular ejection fraction with CIN in different definition

\begin{tabular}{|c|c|c|c|c|c|c|c|c|c|}
\hline \multirow[t]{2}{*}{ Variables } & \multicolumn{3}{|c|}{$\mathrm{CIN}_{0.5 \text { or } 25 \%}$} & \multicolumn{3}{|c|}{$\mathrm{CIN}_{0.3 \text { or } 50 \%}$} & \multicolumn{3}{|c|}{$\mathrm{CIN}_{0.5}$} \\
\hline & OR & $95 \% \mathrm{Cl}$ & $P$-value & OR & $95 \% \mathrm{Cl}$ & P-value & OR & $95 \% \mathrm{Cl}$ & $P$-value \\
\hline $\mathrm{HFpEF}$ & 1 & Reference & - & I & Reference & - & 1 & Reference & - \\
\hline HFrEF vs HFpEF & 1.01 & $0.69-1.74$ & 0.700 & 1.13 & $0.64-1.97$ & 0.676 & 1.10 & $0.53-2.31$ & 0.799 \\
\hline HFmrEF vs HFpEF & 1.31 & $0.87-1.96$ & 0.194 & 1.43 & $0.88-2.33$ & 0.145 & 1.58 & $0.84-2.98$ & 0.158 \\
\hline Age $>75$ years & 1.73 & $1.20-2.49$ & 0.004 & 1.77 & $1.5 \mathrm{I}-2.72$ & 0.010 & 2.07 & $1.18-3.62$ & 0.011 \\
\hline Hypertension & 1.20 & $0.86-1.65$ & 0.280 & 1.52 & $0.98-2.33$ & 0.059 & 1.41 & $0.78-2.56$ & 0.253 \\
\hline DM & 1.08 & $0.77-1.52$ & 0.644 & 0.79 & $0.51-1.22$ & 0.289 & 0.64 & $0.35-1.17$ & 0.146 \\
\hline Renal insufficiency & 0.73 & $0.50-1.06$ & 0.094 & 2.32 & $1.54-3.49$ & $<0.001$ & 3.59 & $2.05-6.27$ & $<0.001$ \\
\hline Advanced HF & 1.54 & I.07-2.22 & 0.019 & 1.63 & $1.03-2.58$ & 0.036 & 2.03 & $1.06-3.89$ & 0.033 \\
\hline Prior MI & 0.90 & $0.55-1.48$ & 0.685 & 0.80 & $0.42-1.53$ & 0.505 & 0.61 & $0.23-1.57$ & 0.303 \\
\hline Emergency PCl & 2.83 & $1.93-4.14$ & $<0.001$ & 2.80 & I.78-4.40 & $<0.001$ & 2.93 & $1.63-5.28$ & $<0.001$ \\
\hline Stains & 0.71 & $0.34-1.47$ & 0.351 & 0.36 & $0.16-0.78$ & 0.009 & 0.23 & $0.09-0.59$ & 0.002 \\
\hline Diuretics & 1.73 & $1.21-2.47$ & 0.003 & 1.80 & I. $17-2.77$ & 0.007 & $\mathrm{I} .4 \mathrm{I}$ & $0.79-2.5$ I & 0.243 \\
\hline $\mathrm{CM}$ volume $>100 \mathrm{~mL}$ & 1.21 & $0.89-1.66$ & 0.223 & 1.36 & $0.91-2.03$ & 0.134 & 1.52 & $0.87-2.65$ & 0.142 \\
\hline Hypotension & 1.28 & $0.68-2.39$ & 0.445 & 1.67 & $0.85-3.31$ & 0.138 & 2.29 & $1.04-5.04$ & 0.04 \\
\hline IABP & 2.45 & $1.44-4.19$ & $<0.001$ & 3.65 & $2.08-6.40$ & $<0.001$ & 3.83 & $|.95-7.5|$ & $<0.001$ \\
\hline
\end{tabular}

Abbreviations: $\mathrm{CIN}$, contrast-induced nephropathy; $\mathrm{OR}$, odds ratio; $\mathrm{Cl}$, confidence interval; $\mathrm{HFpEF}$, heart failure with preserved ejection fraction; $\mathrm{HFrEF}$, heart failure with reduced ejection fraction; HFmrEF, heart failure with mid-range ejection fraction; DM, diabetes mellitus; HF, heart failure; MI, myocardial infarction; PCl, percutaneous coronary intervention; CM, contrast medium; IABP, intra-aortic balloon pump.

emergency PCI and use of IABP were the independent risk factors for CIN. It is noteworthy that reduced LVEF was an independent predictor of long-term mortality following CAG/PCI.

In recent years, the proportion of patients with $\mathrm{HFpEF}$ has increased significantly, ${ }^{21}$ with a prevalence of $71 \%-74 \%$ being reported in large-cohort studies from Western and

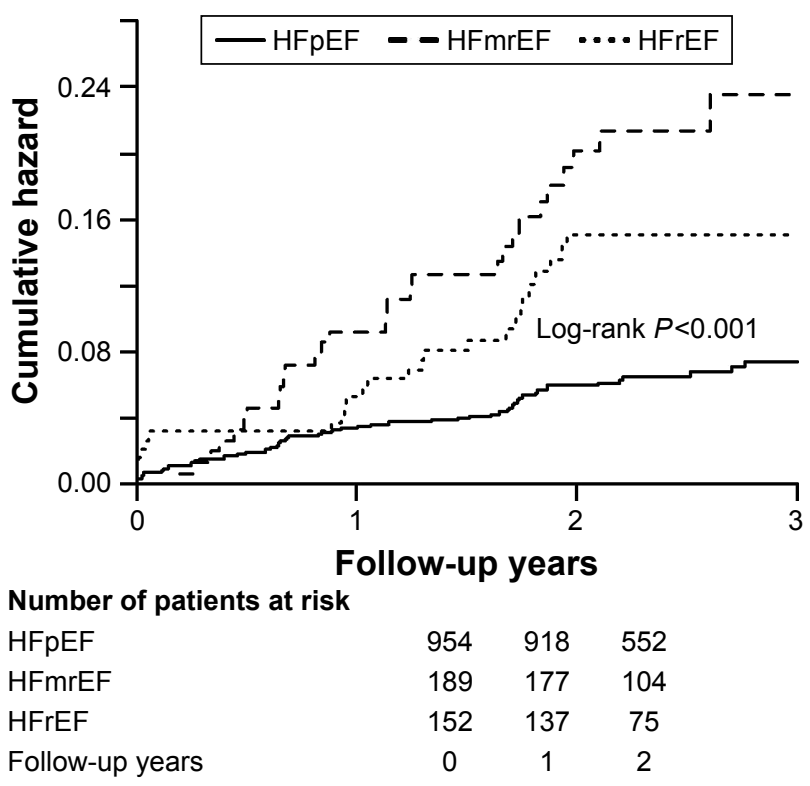

Figure 2 Cumulative rate of all-cause mortality during the follow-up in patients with HFrEF, HFmrEF and HFpEF.

Abbreviations: $\mathrm{HFrEF}$, heart failure with reduced ejection fraction; $\mathrm{HFmrEF}$, heart failure with mid-range ejection fraction; HFpEF, heart failure with preserved ejection fraction.
Asians countries. ${ }^{22-24}$ Additionally, myocardial ischemia has been demonstrated as the major etiology of HF. ${ }^{25,26}$ However, the incidence of HFpEF among these patients following CAG/PCI has not been analyzed. As observed in our analysis, the incidence of HFpEF was highest in the study population (72.98\%), which was similar to the prior analyses. The high prevalence of HFpEF suggests that it should be given high priority in risk assessment.

Characteristics of HFmrEF were demonstrated to be intermediate between those of HFrEF and HFpEF. ${ }^{27}$ Similar results were found in the patients with $\mathrm{HF}$ following $\mathrm{CAG} /$

Table 4 Association between left ventricular ejection fraction and long-term mortality

\begin{tabular}{|c|c|c|c|c|c|c|}
\hline \multirow[t]{2}{*}{ Variables } & \multicolumn{3}{|c|}{ Univariate } & \multicolumn{3}{|c|}{ Multivariate } \\
\hline & HR & $95 \% \mathrm{Cl}$ & $P$-value & HR & $95 \% \mathrm{Cl}$ & $P$-value \\
\hline HFpEF & 1 & Reference & - & I & Reference & - \\
\hline HFrEF vs HFpEF & 3.42 & $2.9-5.35$ & $<0.001$ & 2.88 & $1.77-4.69$ & $<0.001$ \\
\hline HFmrEF vs HFpEF & 2.22 & $1.39-3.55$ & $<0.001$ & 1.55 & $0.95-2.53$ & 0.079 \\
\hline Age $>75$ years & 2.52 & $1.69-3.76$ & $<0.001$ & 1.76 & I.14-2.72 & 0.011 \\
\hline Hypertension & 1.00 & $0.68-1.46$ & 0.998 & 0.91 & $0.61-1.37$ & 0.66 \\
\hline Renal insufficiency & 4.55 & $3.14-6.60$ & $<0.001$ & 3.04 & $2.02-4.56$ & $<0.001$ \\
\hline DM & 1.61 & I.09-2.37 & 0.017 & 1.38 & $0.93-2.07$ & 0.113 \\
\hline IABP & 4.66 & $2.90-7.49$ & $<0.001$ & 2.00 & I.18-3.38 & 0.01 \\
\hline Advanced HF & 2.19 & $1.5 \mathrm{I}-3.17$ & $<0.001$ & 0.83 & $0.53-1.30$ & 0.409 \\
\hline Anemia & 2.04 & $1.4 I-2.96$ & $<0.001$ & 1.46 & $0.99-2.17$ & 0.056 \\
\hline Emergency $\mathrm{PCl}$ & 3.35 & $2.25-5.00$ & $<0.001$ & 2.49 & $1.55-4.00$ & $<0.001$ \\
\hline
\end{tabular}

Abbreviations: $\mathrm{HR}$, hazard ratio; $\mathrm{Cl}$, confidence interval; $\mathrm{HFpEF}$, heart failure with preserved ejection fraction; HFrEF, heart failure with reduced ejection fraction; HFmrEF, heart failure with mid-range ejection fraction; DM, diabetes mellitus; $\mathrm{IABP}$, intra-aortic balloon pump; $\mathrm{HF}$, heart failure; $\mathrm{PCl}$, percutaneous coronary intervention. 


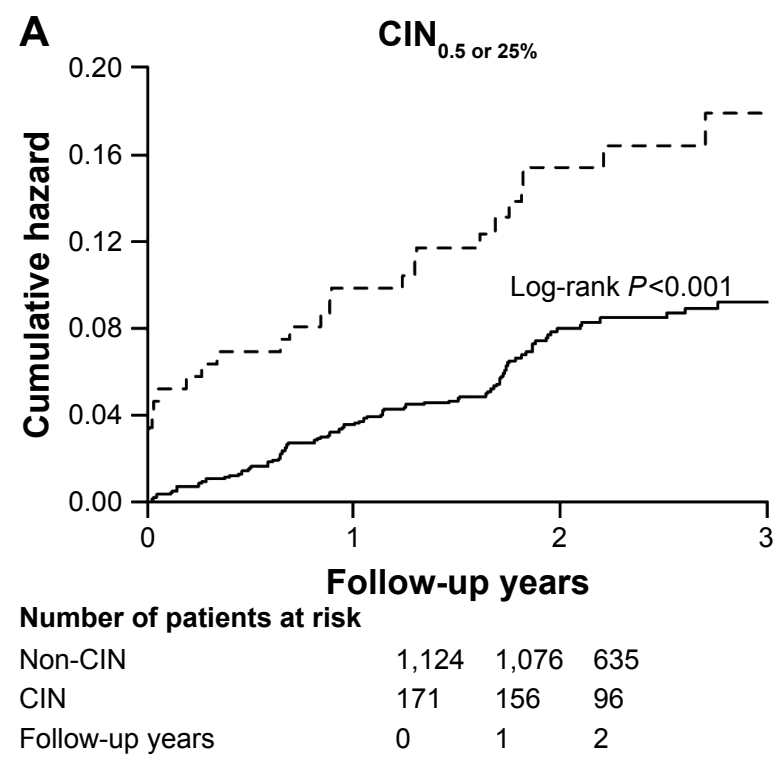

Follow-up years

$\begin{array}{lll}171 & 156 & 96 \\ 0 & 1 & 2\end{array}$

C

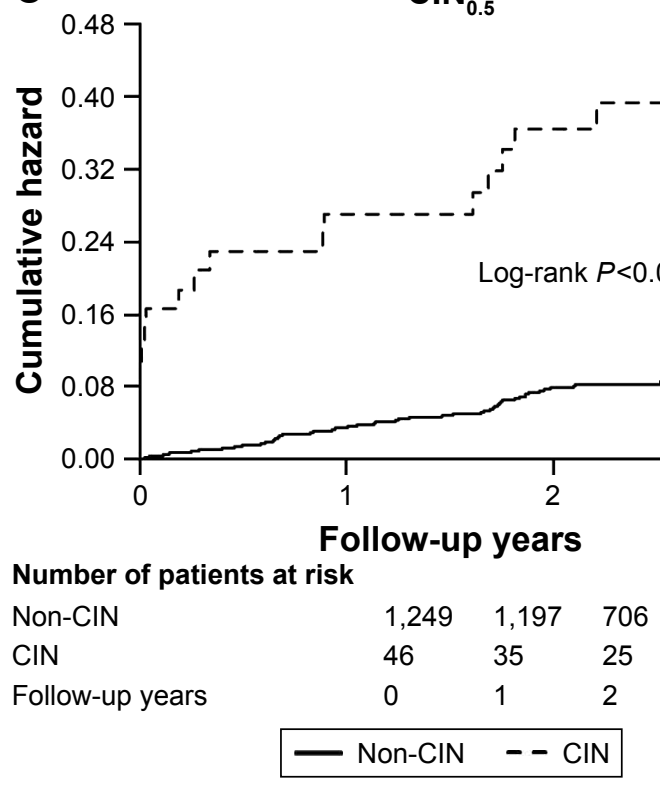

B $\quad$ CIN $_{0.3}$ or $50 \%$

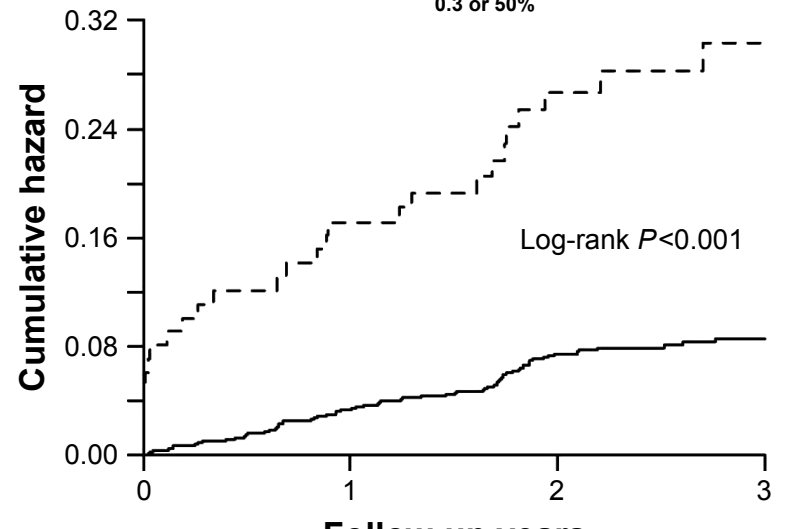

Number of patients at risk

$\begin{array}{llll}\text { Non-CIN } & 1,198 & 1,150 & 679 \\ \text { CIN } & 97 & 82 & 52 \\ \text { Follow-up years } & 0 & 1 & 2\end{array}$

Figure 3 Cumulative all-cause mortality of $\mathrm{CIN}$ and Non-CIN within the definition of $(\mathbf{A}) \mathrm{CIN}_{0.5 \text { or } 25 \%},(\mathbf{B}) \mathrm{CIN}_{0.3 \text { or } 50 \%},(\mathbf{C}) \mathrm{CIN}_{0.5}$. Abbreviation: $\mathrm{CIN}$, contrast-induced nephropathy.

PCI. Our present study indicated that HFmrEF patients were closer to the HFrEF patients in terms of use of diuretics and IABP and presence of comorbidities, such as advanced HF, renal insufficiency and hypotension, but closer to the HFpEF in terms of use of statins, all of which have been demonstrated as contributing factors for CIN. ${ }^{19,28}$ Moreover, patients with HFmrEF were more likely to undergo emergency PCI than other groups. Based on those characteristics, the incidence of CIN was highest in this particular population. In recent years, CIN has been reported as the third most common cause of hospital-acquired renal failure. ${ }^{3}$ Therefore, effective pre-procedural identification of patients at high risk of $\mathrm{CIN}$ is vital.
LVEF is the most widely used parameter to evaluate cardiac functions associated with hemodynamic instability, and consequently causes inadequate renal perfusion. However, the association between LVEF and CIN still remains controversial. An observational study by Shacham et al ${ }^{11}$ included 386 patients undergoing PCI and found that patients with worsened LVEF had significantly higher rate of CIN compared with those with $\mathrm{LVEF} \geq 45 \%$ (14.4\% vs $5.7 \%$; $P=0.02)$. Moreover, worsened LVEF was an independent predictor of CIN. Similar results were found in another extensive cohort study, and a risk score of CIN was named AGEF, including advanced age, depressed LVEF and reduced eGFR. ${ }^{12,29}$ However, studies conducted by Kurtul 
et $\mathrm{al}^{13}$ and Barbieri et $\mathrm{al}^{14}$ showed an opposite effect after adjusting for several confounders. As observed in all the above-mentioned studies, only a small number of patients with HF were included, and consequently, those studies were unable to analyze the association between LVEF and CIN . Furthermore, HF, as an important risk factor of $\mathrm{CIN}^{8,19}$ was not included in the multivariate analysis. In contrast, our study included sufficient patients with HF and adjusted for the potential confounders to investigate the association of LVEF with CIN following CAG/PCI.

Previous studies indicated that the incidence of CIN in those with segment elevation myocardial infarction after PCI to be ranged from $10 \%$ to $20 \%$. The potential factors such as impaired hemodynamic stability, large CM dose and insufficient prophylactic hydration led to higher risk of CIN in this particular group. In addition, inflammatory response and neurohumoral factors were also involved in this progress. ${ }^{30}$ Therefore, emergency PCI was significantly and independently related to the risk of CIN. ${ }^{31,32}$ Recently, Duan et al ${ }^{13}$ developed a simple model for early prediction of CIN, which indicated that emergency PCI was a significant influencing factor in this model. Similarly, emergency PCI increased the risk of CIN in our analysis. Therefore, more prophylactic measures and attention should be paid in this particular population.

The physiopathology of CIN remains poorly understood. Nevertheless, hemodynamic deterioration plays a significant role in the process. Worsened cardiac function contributes to the hemodynamic instability, which reduces effective renal blood flow, consequently trigging renin-angiotensin, activating sympathetic nervous system and increasing inflammatory factors and oxygen radical levels, all of which contribute to the development of CIN. ${ }^{34}$ Therefore, among the eight variables from a classical risk assessment model for CIN, three (hypotension, advanced HF and use of IABP) are directly reflecting worsened cardiac function. ${ }^{8}$ In addition, a high NYHA class reflects not only advanced HF but also adverse hemodynamic parameters ${ }^{35}$ which accelerate the renal hypoperfusion and potentiate CIN. Therefore, it is likely that advanced HF plays an important role in the development of CIN in patients with HF.

Furthermore, previous studies suggested that patients with HFrEF experienced higher mortality compared to those with HFpEF, whereas others have indicated similar outcomes among the groups. ${ }^{36-38}$ The marked disparity in long-term prognosis may contribute to the different inclusion criteria and various cut-offs of LVEF to define the type of HF. According to the classification of HF from guideline, ${ }^{10}$ our data demonstrated that HFrEF in patients increased the risk of all-cause mortality. Therefore, early identification of patients at high risk of mortality may assist in directing treatment.

\section{Limitations}

There are several limitations in this study. First, this was a prospective, observational and a single-center study. Therefore, the risk of bias cannot be ruled out, although we attempted to adjust for the confounding factors. Therefore, large-scale multicenter clinical trials are needed before these conclusions can be applied elsewhere. Second, variation in measurement times may lead to missed post-procedure peak levels of creatinine and may underestimate the true incidence of contrastinduced acute kidney injury. Third, as the study was limited to patients with $\mathrm{HF}$, we were unable to extend the results to patients without HF. Fourth, the diagnosis of HF was based on the clinical evaluation, which has limited reliability.

\section{Conclusion}

Our data indicated that in patients with HF, not worsened LVEF but advanced HF was significantly associated with an increased risk of CIN following CAG/PCI. In addition, the reduced LVEF (HFrEF group) was an independent predictor of long-term mortality. The predictive value of worsened LVEF and advanced HF for CIN and mortality following cardiac catheterization needs to be investigated in patients with HF in large multicenter clinical trials.

\section{Acknowledgments}

This work was supported by Guangdong Provincial Cardiovascular Clinical Medicine Research Fund (grant number: 2009X41, awarded to Yong Liu and Ning Tan), Science and Technology Planning Project of Guangdong Province (PRECOMIN study by Yong Liu in 2011; and study grant number 2008A030201002, awarded to Ji-yan Chen) and Guangdong Cardiovascular Institute. This study was also supported by Progress of Science and Technology Project in Guangdong Province (grant numbers: 2013b031800025, 2016b020215130) and Cardiovascular Research Foundation Project of Chinese Medical Doctor Association (grant number: SCRFCMDA201216).

\section{Author contributions}

KW, NT and YL conceived and designed the study and helped to draft the manuscript. KW, HLL and WJB carried out the database search, and SQC performed the statistical analysis. SMSI revised the manuscript critically. JYC performed the data collection and extraction and arrangement. NT and YL approved the final version of the manuscript. 
All authors contributed toward data analysis, drafting and critically revising the paper and agree to be accountable for all aspects of the work.

\section{Disclosure}

The authors report no conflicts of interest in this work.

\section{References}

1. Roffi M, Patrono C, Collet JP, et al; Management of Acute Coronary Syndromes in Patients Presenting without Persistent ST-Segment Elevation of the European Society of Cardiology. 2015 ESC Guidelines for the management of acute coronary syndromes in patients presenting without persistent ST-segment elevation: Task Force for the Management of Acute Coronary Syndromes in Patients Presenting without Persistent ST-Segment Elevation of the European Society of Cardiology (ESC). Eur Heart J. 2016;37(3):267-315.

2. Tsai TT, Patel UD, Chang TI, et al. Contemporary incidence, predictors, and outcomes of acute kidney injury in patients undergoing percutaneous coronary interventions: insights from the NCDR Cath-PCI registry. JACC Cardiovasc Interv. 2014;7(1):1-9.

3. Aurelio A, Durante A. Contrast-induced nephropathy in percutaneous coronary interventions: pathogenesis, risk factors, outcome, prevention and treatment. Cardiology. 2014;128(1):62-72.

4. Stacul F. Reducing the risks for contrast-induced nephropathy. Cardiovasc Intervent Radiol. 2005;28 Suppl 2:S12-S18.

5. Reyes EB, Ha JW, Firdaus I, et al. Heart failure across Asia: same healthcare burden but differences in organization of care. Int J Cardiol. 2016;223:163-167.

6. Ronco C, McCullough P, Anker SD, et al; Acute Dialysis Quality Initiative (ADQI) consensus group. Cardio-renal syndromes: report from the consensus conference of the Acute Dialysis Quality Initiative. Eur Heart J. 2010;31(6):703-711.

7. Rosenstock JL, Gilles E, Geller AB, et al. Impact of heart failure on the incidence of contrast-induced nephropathy in patients with chronic kidney disease. Int Urol Nephrol. 2010;42(4):1049-1054.

8. Mehran R, Aymong ED, Nikolsky E, et al. A simple risk score for prediction of contrast-induced nephropathy after percutaneous coronary intervention: development and initial validation. J Am Coll Cardiol. 2004; 44(7):1393-1399.

9. Rihal CS, Textor SC, Grill DE, et al. Incidence and prognostic importance of acute renal failure after percutaneous coronary intervention. Circulation. 2002;105(19):2259-2264.

10. Ponikowski P, Voors AA, Anker SD, et al; Authors/Task Force Members. 2016 ESC Guidelines for the diagnosis and treatment of acute and chronic heart failure: The Task Force for the diagnosis and treatment of acute and chronic heart failure of the European Society of Cardiology (ESC) Developed with the special contribution of the Heart Failure Association (HFA) of the ESC. Eur Heart J. 2016;37(27):2129-2200.

11. Shacham Y, Leshem-Rubinow E, Gal-Oz A, et al. Association of left ventricular function and acute kidney injury among ST-elevation myocardial infarction patients treated by primary percutaneous intervention. Am J Cardiol. 2015;115(3):293-297.

12. Flint N, Kaufman N, Gal-Oz A, et al. Echocardiographic correlates of left ventricular filling pressures and acute cardio-renal syndrome in ST segment elevation myocardial infarction patients. Clin Res Cardiol. 2017; 106(2):120-126.

13. Kurtul A, Duran M, Yarlioglues M, et al. Association between $\mathrm{N}$-terminal pro-brain natriuretic peptide levels and contrast-induced nephropathy in patients undergoing percutaneous coronary intervention for acute coronary syndrome. Clin Cardiol. 2014;37(8):485-492.

14. Barbieri L, Verdoia M, Nardin M, Marino P, Suryapranata H, De Luca G; Novara Atherosclerosis Study Group (NAS). Gender difference in the risk of contrast-induced nephropathy in patients undergoing coronary angiography or percutaneous coronary intervention. Angiology. 2017; 68(6):542-546.
15. Levey AS, Bosch JP, Lewis JB, Greene T, Rogers N, Roth D. A more accurate method to estimate glomerular filtration rate from serum creatinine: a new prediction equation. Modification of Diet in Renal Disease Study Group. Ann Intern Med. 1999;130(6):461-470.

16. Courtney AE, Maxwell AP, Fogarty DG. Using estimated glomerular filtration rate (eGFR) to help manage patients with chronic kidney disease. Ulster Med J. 2007;76(3):154-156.

17. Metra M, Ponikowski P, Dickstein K, et al; Heart Failure Association of the European Society of Cardiology. Advanced chronic heart failure: a position statement from the Study Group on Advanced Heart Failure of the Heart Failure Association of the European Society of Cardiology. Eur J Heart Fail. 2007;9(6-7):684-694.

18. Killip T 3rd, Kimball JT. Treatment of myocardial infarction in a coronary care unit. A two year experience with 250 patients. Am J Cardiol. 1967;20(4):457-464.

19. Stacul F, van der Molen AJ, Reimer P, et al; Contrast Media Safety Committee of European Society of Urogenital Radiology (ESUR). Contrast induced nephropathy: updated ESUR Contrast Media Safety Committee guidelines. Eur Radiol. 2011;21(12):2527-2541.

20. Zeng X, McMahon GM, Brunelli SM, Bates DW, Waikar SS. Incidence, outcomes, and comparisons across definitions of AKI in hospitalized individuals. Clin J Am Soc Nephrol. 2014;9(1):12-20.

21. Owan TE, Hodge DO, Herges RM, Jacobsen SJ, Roger VL, Redfield MM. Trends in prevalence and outcome of heart failure with preserved ejection fraction. $N$ Engl J Med. 2006;355(3):251-259.

22. Hogg K, Swedberg K, McMurray J. Heart failure with preserved left ventricular systolic function: epidemiology, clinical characteristics, and prognosis. J Am Coll Cardiol. 2004;43(3):317-327.

23. Lam CS, Donal E, Kraigher-Krainer E, Vasan RS. Epidemiology and clinical course of heart failure with preserved ejection fraction. Eur J Heart Fail. 2011;13(1):18-28.

24. Kaneko H, Suzuki S, Yajima J, et al. Clinical characteristics and longterm clinical outcomes of Japanese heart failure patients with preserved versus reduced left ventricular ejection fraction: a prospective cohort of Shinken Database 2004-2011. J Cardiol. 2013;62(2):102-109.

25. Cowie MR, Wood DA, Coats AJ, et al. Incidence and aetiology of heart failure; a population-based study. Eur Heart J. 1999;20(6):421-428.

26. Lam CS, Teng TK, Tay WT, et al. Regional and ethnic differences among patients with heart failure in Asia: the Asian sudden cardiac death in heart failure registry. Eur Heart J. 2016;37(41):3141-3153.

27. Lam CS, Solomon SD. The middle child in heart failure: heart failure with mid-range ejection fraction (40-50\%). Eur J Heart Fail. 2014;16(10):1049-1055.

28. Zhang MM, Lv QZ, Li XY. Drug effects and clinical investigations for contrast-induced nephropathy after coronary angiography or percutaneous coronary intervention in patients with diabetes. Am J Ther. Epub 2015 Aug 24.

29. Andò G, Morabito G, de Gregorio C, Trio O, Saporito F, Oreto G. Age, glomerular filtration rate, ejection fraction, and the AGEF score predict contrast-induced nephropathy in patients with acute myocardial infarction undergoing primary percutaneous coronary intervention. Catheter Cardiovasc Interv. 2013;82(6):878-885.

30. Guerchicoff A, Stone GW, Mehran R, et al. Analysis of biomarkers for risk of acute kidney injury after primary angioplasty for acute STsegment elevation myocardial infarction: results of the HORIZONSAMI trial. Catheter Cardiovasc Interv. 2015;85(3):335-342.

31. Tan N, Liu Y, Zhou YL, et al. Contrast medium volume to creatinine clearance ratio: a predictor of contrast-induced nephropathy in the first 72 hours following percutaneous coronary intervention. Catheter Cardiovasc Interv. 2012;79(1):70-75.

32. Fu N, Li X, Yang S, et al. Risk score for the prediction of contrastinduced nephropathy in elderly patients undergoing percutaneous coronary intervention. Angiology. 2013;64(3):188-194.

33. Duan C, Cao Y, Liu Y, et al. A new preprocedure risk score for predicting contrast-induced acute kidney injury. Can J Cardiol. 2017;33(6):714-723.

34. Azzalini L, Spagnoli V, Ly HQ. Contrast-induced nephropathy: from pathophysiology to preventive strategies. Can J Cardiol. 2016;32(2): 247-255. 
35. Ahmed A, Aronow WS, Fleg JL. Higher New York Heart Association classes and increased mortality and hospitalization in patients with heart failure and preserved left ventricular function. Am Heart J. 2006;151(2):444 450.

36. MacDonald MR, Wee PP, Cao Y, et al. Comparison of characteristics and outcomes of heart failure patients with preserved versus reduced ejection fraction in a multiethnic Southeast Asian cohort. Am J Cardiol. 2016;118(8):1233-1238.
37. Lund LH, Donal E, Oger E, et al; KaRen Investigators. Association between cardiovascular vs. non-cardiovascular co-morbidities and outcomes in heart failure with preserved ejection fraction. Eur J Heart Fail. 2014;16(9):992-1001.

38. Coles AH, Fisher K, Darling C, et al. Long-term survival for patients with acute decompensated heart failure according to ejection fraction findings. Am J Cardiol. 2014;114(6):862-868.

\section{Publish your work in this journal}

Therapeutics and Clinical Risk Management is an international, peerreviewed journal of clinical therapeutics and risk management, focusing on concise rapid reporting of clinical studies in all therapeutic areas outcomes, safety, and programs for the effective, safe, and sustained use of medicines. This journal is indexed on PubMed Central, CAS,
EMBase, Scopus and the Elsevier Bibliographic databases. The manuscript management system is completely online and includes a very quick and fair peer-review system, which is all easy to use. Visit http://www.dovepress.com/testimonials.php to read real quotes from published authors.

Submit your manuscript here: http://www.dovepress.com/therapeutics-and-clinical-risk-management-journal 